\title{
The Westerbork SINGS survey
}

\section{Overview and image atlas}

\author{
R. Braun ${ }^{1}$, T. A. Oosterloo ${ }^{1}$, R. Morganti ${ }^{1}$, U. Klein ${ }^{2}$, and R. Beck ${ }^{3}$ \\ 1 ASTRON, PO Box 2, 7990 AA Dwingeloo, The Netherlands \\ e-mail: braun@astron.nl \\ 2 Argelander-Institut für Astronomie, Radio Astronomy Department, Auf dem Hügel 71, 53121 Bonn, Germany \\ 3 Max-Planck-Institut für Radioastronomie, Auf dem Hügel 69, 53121 Bonn, Germany
}

Received 24 July 2006 / Accepted 23 September 2006

\section{ABSTRACT}

\begin{abstract}
We have obtained moderately deep radio continuum imaging at 18 and $22 \mathrm{~cm}$ with the Westerbork array of 34 nearby galaxies drawn from the Spitzer SINGS and Starburst samples to enable complimentary analysis. The sub-sample have an optical major axis diameter in excess of 5 arcmin and are North of Declination 12.5. Sub-sample galaxies span a very wide range of morphological types and star formation rates. Resolved detection was possible for every galaxy. This constitutes a first time detection at GHz radio frequencies for about half of the sample. Analysis of both total intensity and polarization properties of the sample will be published in companion papers. Both the $\mathrm{HI}$ and $\mathrm{OH}$ main-lines of the target galaxies were within the observed band-pass, albeit with only coarse velocity resolution. Only two low mass elliptical galaxies were undetected in H I. Four of the sub-sample galaxies were detected in $\mathrm{OH}$ main-line absorption, including two new detections. The results are presented in the form of an image atlas for which a standard transfer function and image size are used throughout and whereby the radio continuum, DSS optical and integrated H I are displayed side-by-side. Continuum and $\mathrm{H}$ l line photometry are tabulated for all targets.
\end{abstract}

Key words. radio continuum: galaxies - galaxies: general - galaxies: ISM - radio lines: galaxies

\section{Introduction}

An understanding of the star formation process is critical to a wide range of astrophysical questions, from the phase and energy balance in the local ISM, to the circumstances surrounding the universal peak of star formation and merger activity that apparently typify the early universe. The traditional global tracers of current star formation in galaxies, like $\mathrm{H} \alpha$ imaging, have led to some empirical descriptions of how SF occurs (e.g. Kennicutt 1988). However, given the complexity of the many competing processes ocurring in a galactic disk, it is clear that a coordinated effort, including multiple ISM tracers, will be needed to make more substantial progress. Just such an initiative is currently underway with the Spitzer Nearby Galaxy Survey (SINGS, Kennicutt et al. 2003). This Spitzer Legacy program provides pixel-resolved SED data from the visible to $160 \mu \mathrm{m}$ for a sample of 75 galaxies nearer than $30 \mathrm{Mpc}$ that span the largest possible range of Hubble types and (to a lesser extent) SFR's. In addition to the Spitzer data, the SINGS program will make a comprehensive set of broad-band optical BVRIJHK, narrowband $\mathrm{H} \alpha$, as well as UV, CO and (in some cases) HI data available to the general public.

An important supplement to the SINGS program is sensitive radio continuum imaging of the sample galaxies. In this paper we describe the acquisition of such data with the Westerbork Synthesis Radio Telescope (WSRT) for a subsample of thirty SINGS galaxies, supplemented with a subsample of four Starburst galaxies. Radio continuum data has been acquired in the $20 \mathrm{~cm}$ band which reaches a $3 \sigma$ Emission Measure $(E M)$ sensitivity of $75 \mathrm{pc} \mathrm{cm}^{-6}$ for emission filling the $1 \mathrm{kpc}$ beam (at the average galaxy distance of $10 \mathrm{Mpc}$ ). For comparison, the Diffuse Ionized Gas which fills the disks of spiral galaxies has a typical peak $E M$ determined from $\mathrm{H} \alpha$ imaging of about $100 \mathrm{pc} \mathrm{cm}^{-6}$, while discrete $\mathrm{H}$ II region complexes reach $E M$ 's of $10^{3}-10^{4} \mathrm{pc} \mathrm{cm}^{-6}$ (e.g. Greenawalt et al. 1998). Our continuum sensitivity is thus sufficient to detect both diffuse and discrete regions of thermal emission within our target galaxy disks in addition to the much brighter non-thermal component which typically accounts for about $75 \%$ of the total galaxy flux at these frequencies.

Amongst the various scientific goals which will be addressed with our newly acquired data are a resolved study of the FIR-radio correlation (for which the first analysis paper has just appeared in Murphy et al. 2006), a systematic study of galaxy magnetic fields and an unbiased survey of $\mathrm{HI}$ and $\mathrm{OH}$ emission in both the target galaxies as well as their extended environment. These and other topics will addressed in forthcoming papers.

The current paper is organized as follows. We describe the sample definition, observations and reduction in Sect. 2 and present the results in Sect. 3. Digital images will be made available from the Spitzer SINGS web site at http://data.spitzer.caltech.edu/popular/sings/

\section{Observations and reduction}

The SINGS sample (Kennicutt et al. 2003) includes 75 "normal" galaxies within $30 \mathrm{Mpc}$ that span (as uniformly as possible) Hubble types from Irregular to Elliptical and star formation rates from less than 0.001 to more than $10 M_{\odot} \mathrm{yr}^{-1}$. We define our sub-sample of SINGS galaxies by choosing those North of Declination 12.5 and for which $\mathrm{D}_{25}$ (the optical $B$ band 
Table 1. Galaxy observations and data attributes.

\begin{tabular}{|c|c|c|c|c|c|c|c|}
\hline Name & Observation dates & $\begin{array}{l}18 \mathrm{~cm} \text { beam } \\
\mathrm{NS} \times E W\left({ }^{\prime \prime}\right)\end{array}$ & $\begin{array}{c}18 \mathrm{~cm} \mathrm{rms} \\
(\mu \mathrm{Jy} / \text { Beam })\end{array}$ & $\begin{array}{l}22 \mathrm{~cm} \text { beam } \\
\mathrm{NS} \times E W\left({ }^{\prime \prime}\right)\end{array}$ & $\begin{array}{c}22 \mathrm{~cm} \mathrm{rms} \\
(\mu \mathrm{Jy} / \mathrm{Beam})\end{array}$ & $\begin{array}{c}\text { HI beam } \\
\mathrm{NS} \times E W\left({ }^{\prime \prime}\right)\end{array}$ & $\begin{array}{c}\text { HI rms } \\
(\mu \mathrm{Jy} / \text { Beam@625 kHz })\end{array}$ \\
\hline Holmberg II & $2003 / 03 / 07$ & $10.5 \times 10.0$ & 38 & $13.0 \times 12.5$ & 27 & $24.8 \times 17.6$ & 140 \\
\hline IC 2574 & $2003 / 03 / 23$ & $10.5 \times 10.0$ & 36 & $13.0 \times 12.5$ & 24 & $20.1 \times 19.1$ & 120 \\
\hline NGC 0628 & $2003 / 03 / 09$ & $36.5 \times 10.0$ & 40 & $45.5 \times 12.5$ & 29 & $62.5 \times 16.9$ & 160 \\
\hline NGC 0925 & $2003 / 03 / 22$ & $18.0 \times 10.0$ & 36 & $22.5 \times 12.5$ & 26 & $31.7 \times 19.2$ & 140 \\
\hline NGC 2146 & 2003/03/08 & $10.0 \times 10.0$ & 40 & $12.5 \times 12.5$ & 29 & $19.0 \times 18.4$ & 150 \\
\hline NGC 2403 & $2003 / 04 / 06$ & $10.5 \times 10.0$ & 37 & $13.5 \times 12.5$ & 26 & $20.9 \times 19.6$ & 140 \\
\hline NGC 2841 & $2003 / 03 / 10$ & $12.5 \times 10.0$ & 35 & $16.0 \times 12.5$ & 25 & $24.0 \times 20.1$ & 130 \\
\hline NGC 2903 & $2003 / 03 / 26$ & $27.0 \times 10.0$ & 38 & $34.0 \times 12.5$ & 32 & $45.4 \times 18.3$ & 140 \\
\hline NGC 2976 & $2003 / 03 / 21$ & $10.5 \times 10.0$ & 36 & $13.0 \times 12.5$ & 24 & $20.9 \times 19.7$ & 140 \\
\hline NGC 3031 & $2003 / 04 / 17+05 / 05$ & - & - & $14.5 \times 13.6$ & 24 & - & - \\
\hline NGC 3034 & $2003 / 03 / 16$ & $10.5 \times 10.0$ & 75 & - & - & - & - \\
\hline NGC 3079 & $2003 / 03 / 27$ & $12.0 \times 10.0$ & 40 & $15.0 \times 12.5$ & 34 & $23.1 \times 21.1$ & 170 \\
\hline NGC 3184 & $2003 / 05 / 10$ & $15.0 \times 10.0$ & 36 & $18.5 \times 12.5$ & 25 & $27.2 \times 19.2$ & 140 \\
\hline NGC 3198 & $2003 / 04 / 09$ & $14.0 \times 10.0$ & 37 & $17.5 \times 12.5$ & 27 & $25.6 \times 20.2$ & 150 \\
\hline NGC 3627 & $2003 / 04 / 10$ & $44.0 \times 10.0$ & 46 & $55.5 \times 12.5$ & 42 & $75.5 \times 17.3$ & 170 \\
\hline NGC 3628 & $2003 / 04 / 08$ & $42.5 \times 10.0$ & 43 & $53.0 \times 12.5$ & 32 & $72.0 \times 17.0$ & 160 \\
\hline NGC 3938 & $2003 / 03 / 13$ & $14.0 \times 10.0$ & 36 & $17.5 \times 12.5$ & 26 & $25.2 \times 19.3$ & 140 \\
\hline NGC 4125 & $2003 / 03 / 17$ & $11.0 \times 10.0$ & 107 & $13.5 \times 12.5$ & 27 & $24.1 \times 22.1$ & 150 \\
\hline NGC 4236 & $2003 / 05 / 08$ & $10.5 \times 10.0$ & 42 & $13.0 \times 12.5$ & 24 & $20.6 \times 19.7$ & 140 \\
\hline NGC 4254 & $2003 / 04 / 15$ & $40.0 \times 10.0$ & 44 & $50.0 \times 12.5$ & 34 & $68.6 \times 17.0$ & 160 \\
\hline NGC 4321 & $2003 / 04 / 16$ & $36.5 \times 10.0$ & 42 & $45.5 \times 12.5$ & 31 & $62.6 \times 17.2$ & 180 \\
\hline NGC 4450 & $2003 / 04 / 21$ & $34.0 \times 10.0$ & 39 & $42.5 \times 12.5$ & 27 & $58.1 \times 17.4$ & 170 \\
\hline NGC 4552 & $2003 / 07 / 10$ & $46.0 \times 10.0$ & 90 & $57.5 \times 12.5$ & 35 & $77.6 \times 17.2$ & 160 \\
\hline NGC 4559 & $2003 / 06 / 30$ & $21.0 \times 10.0$ & 53 & $26.5 \times 12.5$ & 26 & $36.3 \times 19.2$ & 150 \\
\hline NGC 4569 & $2003 / 03 / 24$ & $43.5 \times 10.0$ & 73 & $54.5 \times 12.5$ & 26 & $74.1 \times 17.3$ & 150 \\
\hline NGC 4631 & $2003 / 04 / 03$ & $18.5 \times 10.0$ & 40 & $23.0 \times 12.5$ & 27 & $32.1 \times 18.9$ & 150 \\
\hline NGC 4725 & $2003 / 07 / 04$ & $23.0 \times 10.0$ & 43 & $29.0 \times 12.5$ & 27 & $39.1 \times 18.3$ & 180 \\
\hline NGC 4736 & $2003 / 07 / 06$ & $15.0 \times 10.0$ & 56 & $19.0 \times 12.5$ & 32 & $27.5 \times 19.6$ & 140 \\
\hline NGC 4826 & $2003 / 06 / 21$ & $27.0 \times 10.0$ & 142 & $33.5 \times 12.5$ & 28 & $45.1 \times 18.0$ & 150 \\
\hline NGC 5033 & 2003/04/01 & $16.5 \times 10.0$ & 38 & $20.5 \times 12.5$ & 29 & $30.1 \times 19.1$ & 150 \\
\hline NGC 5055 & $2003 / 05 / 06$ & $14.5 \times 10.0$ & 39 & $18.5 \times 12.5$ & 27 & $30.6 \times 16.6$ & 150 \\
\hline NGC 5194 & $2003 / 03 / 25+11 / 23$ & $13.5 \times 10.0$ & 28 & $17.0 \times 12.5$ & 22 & $24.1 \times 19.3$ & 100 \\
\hline NGC 6946 & $2003 / 03 / 23+11 / 22$ & $11.5 \times 10.0$ & 28 & $14.0 \times 12.5$ & 20 & $21.5 \times 19.3$ & 100 \\
\hline NGC 7331 & $2003 / 03 / 30+07 / 09$ & $17.5 \times 10.0$ & 31 & $22.0 \times 12.5$ & 23 & $33.1 \times 17.9$ & 110 \\
\hline
\end{tabular}

isophotal diameter at a brightness of 25 mag per square arcsec) is greater than 5 arcmin. These criteria were used to insure that the angular resolution across the targets would be sufficient to enable resolved study, given the limitations of the WSRT array (an east-west configuration of $2.7 \mathrm{~km}$ extent). The resulting thirty SINGS galaxies were supplemented by applying the same selection criteria to the Spitzer Starburst GTO program of G. Rieke (http://ssc.spitzer.caltech.edu/geninfo/gto/). This yielded four additional targets which extend our sample into the very high star formation rate regime.

Each target was observed for one (and occasionally two) twelve hour integration(s) in the "maxi-short" array configuration, in which shortest East-West baselines of 36, 54, 72 and $90 \mathrm{~m}$ are all measured simultaneously as well as a longest baseline of about $2700 \mathrm{~m}$. Details of observing sessions can be found in Table 1 . The target observations were bracketed by observations of the primary total intensity and polarization calibration sources 3C147 and 3C286, as well as additional calibrators CTD93 and 3C138, yielding an absolute flux density calibration accuracy of better than $5 \%$. The observing frequency was switched every five minutes between two settings (1300 to $1432 \mathrm{MHz}$ and 1631 to $1763 \mathrm{MHz}$ ). Each frequency setting was covered with eight sub-bands of $20 \mathrm{MHz}$ nominal width, but spaced by $16 \mathrm{MHz}$ to provide contiguous, non-attenuated coverage. An effective integration time of 6 (or 12) hours was realized at each frequency setting. All four polarizations products and 64 spectral channels were obtained in each sub-band. After careful editing of incidental radio frequency interference (RFI), external total intensity, band-pass and polarization calibration of the data was performed in the AIPS package.

Subsequently, each field was self-calibrated in Stokes $I, Q$ and $U$ using an imaging pipeline based on the Miriad package. Each of the eight sub-bands for a given frequency setting was first processed and imaged independently; and these were subsequently combined with an inverse variance weighting. Deconvolution of each sub-band image was performed iteratively within a threshold mask based on a spatial smoothing of the previous iterate. The individual frequency channels (of $312.5 \mathrm{kHz}$ width) were gridded during imaging, so that band-width smearing effects were negligible. Before combination, each deconvolved sub-band image was subjected to a linear deconvolution of the Gaussian restoring beam followed by Gaussian tapering to a nominal PSF size as well as a primary beam correction appropriate to the sub-band observing frequency. The final combined image Gaussian PSF parameters and actual rms noise levels are shown in Table 1.

A different reduction strategy was employed for NGC 3031. The diffuse emission from this galaxy was so extended (filling the entire telescope primary beam) that the individual subband images were strongly self-confused. This severely hampered self-calibration and imaging of the individual sub-bands. The best results for this galaxy were obtained by including the data from all 16 sub-bands (both $18 \mathrm{~cm}$ and $22 \mathrm{~cm}$ ) simultaneously in the the self-calibration and imaging process (using the 

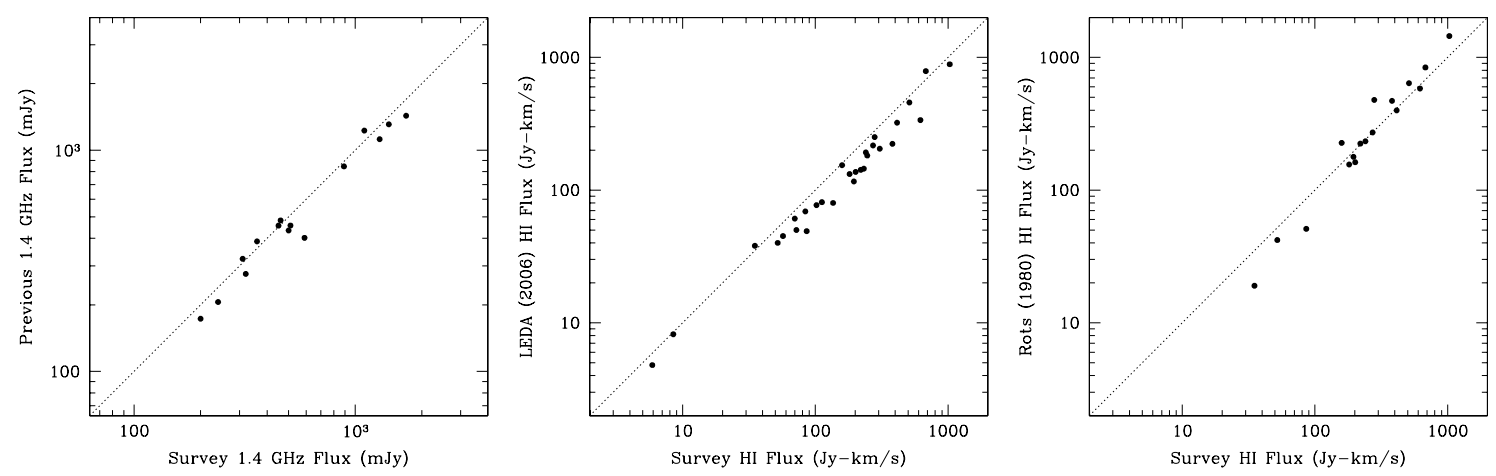

Fig. 1. Radio continuum (left), and neutral hydrogen (center and right) flux comparison of the current survey with previously published values. The comparison data is that of White \& Becker (1992) for the radio continuum and LEDA (http:/leda.univ-lyon1.fr) (center) and Rots (1980) (right) for the neutral hydrogen.

Miriad task mfclean). Consequently, only a single final image (at effective frequency $1589 \mathrm{MHz}$ ) is available for this galaxy.

For the bright starburst galaxy NGC 3034, only the $18 \mathrm{~cm}$ band data could be successfully calibrated and imaged.

As can be seen from Table 1, the noise performance is significantly better for most targets, by about $50 \%$, in the $22 \mathrm{~cm}$ band than in the $18 \mathrm{~cm}$ band. This is partly due to a higher system temperature of the receiving system near $1700 \mathrm{MHz}$ than near $1400 \mathrm{MHz}$, but is also due to higher levels of RFI near $1700 \mathrm{MHz}$. Residual RFI and calibration imperfections account for the variations in final image quality. A few particularly bad examples are the $18 \mathrm{~cm}$ images of NGC 4125 and NGC 4826. The noise performance in the $22 \mathrm{~cm}$ band is in all cases somewhat worse than the theoretical value of $15 \mu \mathrm{Jy}$ (for an ideal $6 \mathrm{~h}$ integration) partly due to the data weighting strategy employed (a semi-uniform weighting using a robustness parameter of -1 in the Miriad task Invert), but also due to source and sidelobe confusion effects in these complex fields. Repeat observations were obtained for those targets which had substantial data loss in the original coverage due to RFI or equipment failures.

The two observing bands were chosen to provide frequency coverage of the neutral hydrogen (at $22 \mathrm{~cm}$ ) and hydroxyl (at $18 \mathrm{~cm}$ ) emission lines at the recession velocities of all targets as well as their extended environment. The $312.5 \mathrm{kHz}$ frequency resolution, corresponding to about $66 \mathrm{~km} \mathrm{~s}^{-1}$ in the $\mathrm{HI}$ line, is rather coarse, while the total velocity coverage of some $27000 \mathrm{~km} \mathrm{~s}^{-1}$, is extremely broad. The expected velocity range of the Hi line due to our own Galaxy and that of each target galaxy was excluded from the continuum imaging described above. An effective continuum model was available for each observed sub-band (as a by-product of the self-calibration step described above). The continuum model was subtracted from the visibility data of each sub-band. The residual data were imaged, hanning smoothed in velocity, deconvolved and subjected to a residual continuum subtraction in the image plane. The resulting line image cubes have the PSF and rms properties listed in Table 1. The PSF dimensions are those that result from a seminatural data weighting (a robustness parameter of +0.5 in the Miriad task Invert). Images of integrated H I emission were generated by summing masked channel images at nominal angular resolution. The mask for each channel was generated by forming a convolved channel image with 120 arsec resolution and including in the mask those regions which exceeded five times the rms level in either the smoothed or the original image. This is a very conservative limit on H I detection; normally a threshold in smoothed images of between 2 and $3 \sigma$ is employed. However, in view of the very coarse intrinsic velocity resolution and semi-natural nominal PSF this criterion was found to be quite effective in practise and eliminated the need for any interactive image blanking. Manual inspection of the channel images, particularly for the two undetected targets did not reveal any significant emission features missed by our masking criterion.

\section{Results}

An overview of the imaging results is given in Figs. 2-10, where radio continuum, optical $R$ band and integrated $\mathrm{H}$ I emission are displayed for our sample galaxies. The same transfer function (as indicated in the figure captions) and standard image size $(28 \times 28$ arcmin) have been used for all galaxies to facilitate comparison. The median was subtracted from each optical DSS image before display. The only minor complication to direct intercomparison is the choice of a fixed scale in flux units (Jy/Beam) rather than surface brightness units in the presentation of the radio continuum images since the beam area varies by almost a factor of five between low Declination and high Declination targets. We comment briefly on each of the targets in Sect. 3.1 below.

The total detected flux density in the $22 \mathrm{~cm}$ continuum band has been determined for each target galaxy and is tabulated in Table 2, excluding NGC 3031 and NGC 3034, for which appropriate $22 \mathrm{~cm}$ images were not available. The aperture radius and flux integration method are indicated in the table for each galaxy. In those cases where no extended disk emission was detected, a simple rectangular "Box" or "Poly"-gon was integrated with the noted effective radius. In a single case (NGC 4125) an elliptical Gaussian was fit to the detected source; method "GFit". In most cases, the diffuse brightness distribution was integrated in concentric circles, whereby a background brightness level was estimated and subtracted from the integral; method "Rad". The background estimate was determined at radii extending $10 \%$ beyond the radius indicated in the table. In those cases where particularly bright background radio sources were present in the field, such sources were first interactively blanked from the image, before integration in concentric circles as above; method "Blk/Rad". The error estimates in the table reflect the variation in total flux that result from alternate choices of the integation radius. Although all of these values either agree with or slightly exceed (by up to 20\%) current estimates in the literature (White \& Becker 1992) they must still be regarded as lower limits, since the brightness distribution declines so smoothly into the noise floor. Our measured fluxes are compared with those of White \& Becker in Fig. 1. About half of the entries in our table 

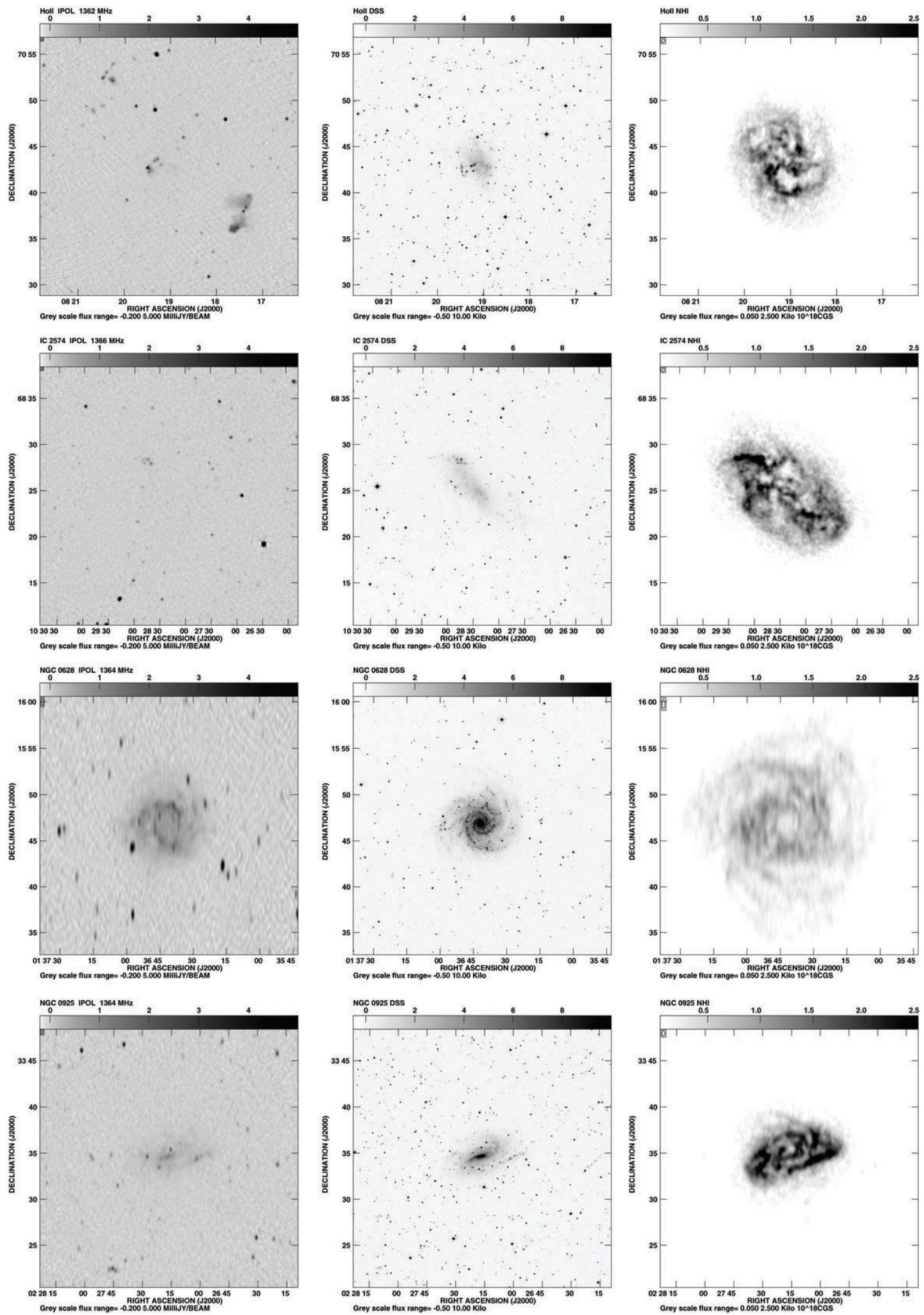

Fig. 2. Radio continuum (left), optical POSS-II Red (center) and neutral hydrogen column density (right) for the four indicated galaxies. A square root transfer function is used for the radio continuum image and a linear one for the optical (in densitometer counts) and $\mathrm{HI}$ images.

correspond to first time detections of continuum emission from these galaxies at $\mathrm{GHz}$ radio frequencies.

The integrated H I line flux is also listed in Table 2 for each galaxy together with the peak observed column density. The error estimates in total HI line flux include uncertainties due to our conservative masking criterion. In view of the very low recession velocites of NGC 3031 and NGC 3034, the H I emission from these galaxies was strongly confused with that of our Galaxy and is not shown or tabulated. The only non-detections in H I are NGC 4125 and NGC 4552 with the indicated $3 \sigma$ upper limits. Our measured Hi fluxes are compared with those in the literature in Fig. 1, where we have taken the LEDA database 

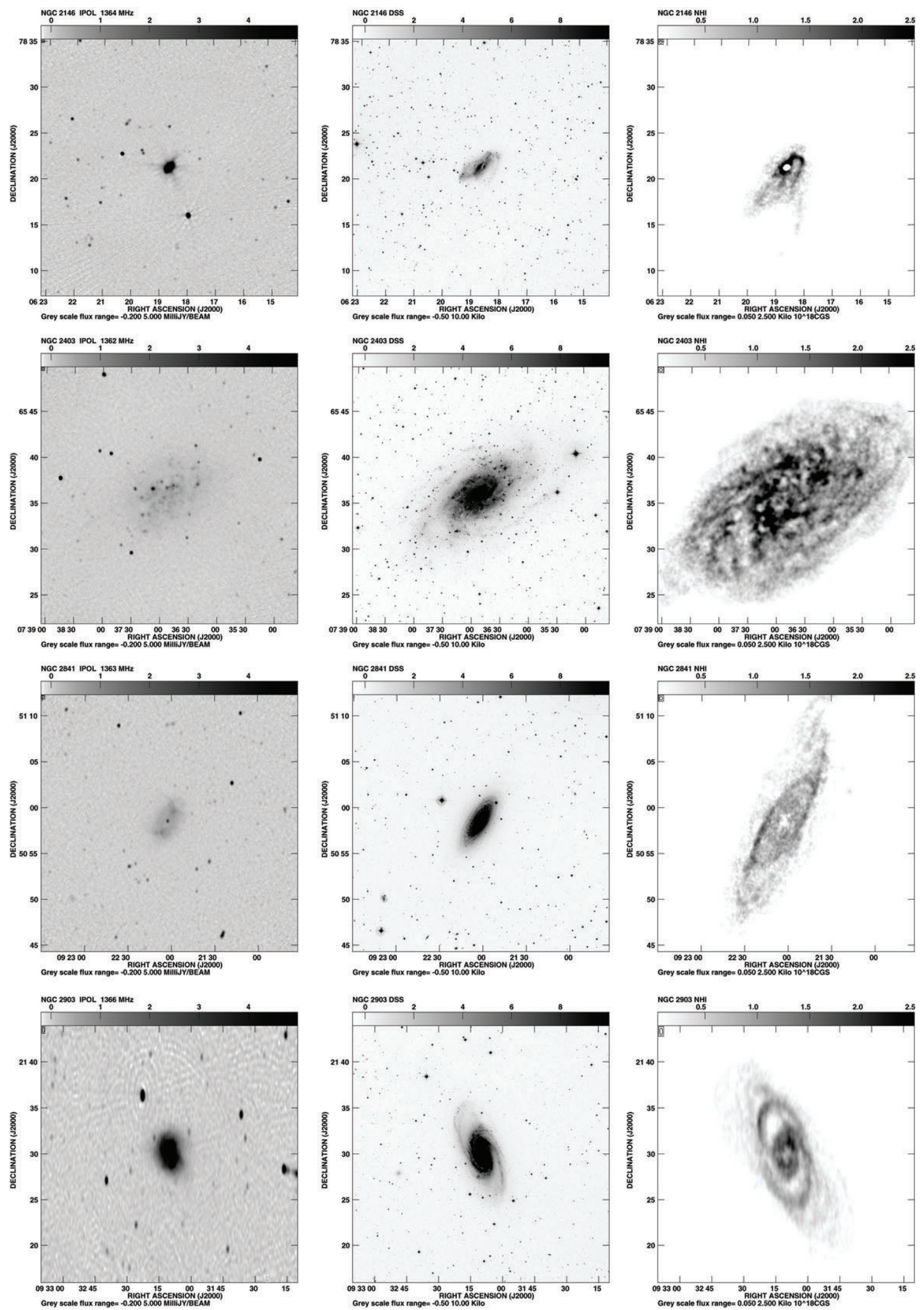

Fig. 3. Radio continuum (left), optical POSS-II Red (center) and neutral hydrogen column density (right) for the four indicated galaxies. A square root transfer function is used for the radio continuum image and a linear one for the optical (in densitometer counts) and $\mathrm{HI}$ images.

entries (http:/leda.univ-lyon1.fr) from June 2006 and those of Rots (1980) for comparison. As can be seen in the figure, our fluxes either agree with, or exceed (by as much as a factor of 1.8) those tabulated in LEDA. Comparison with the Rots values indicates that only the two galaxies of largest angular size in our sample (NGC 2403 and 5055) have significantly low detected fluxes (by factors of 1.4 and 1.7). There is also a discrepancy of a factor of 1.4 in the NGC 5194 field, due to the extended tidal debris and companions in this region. Smaller underestimates (by a factor of 1.2) are present in the cases of NGC 628 and 6946. We recognize that our flux values must be considered as lower limits, given our finite sensitivity and 

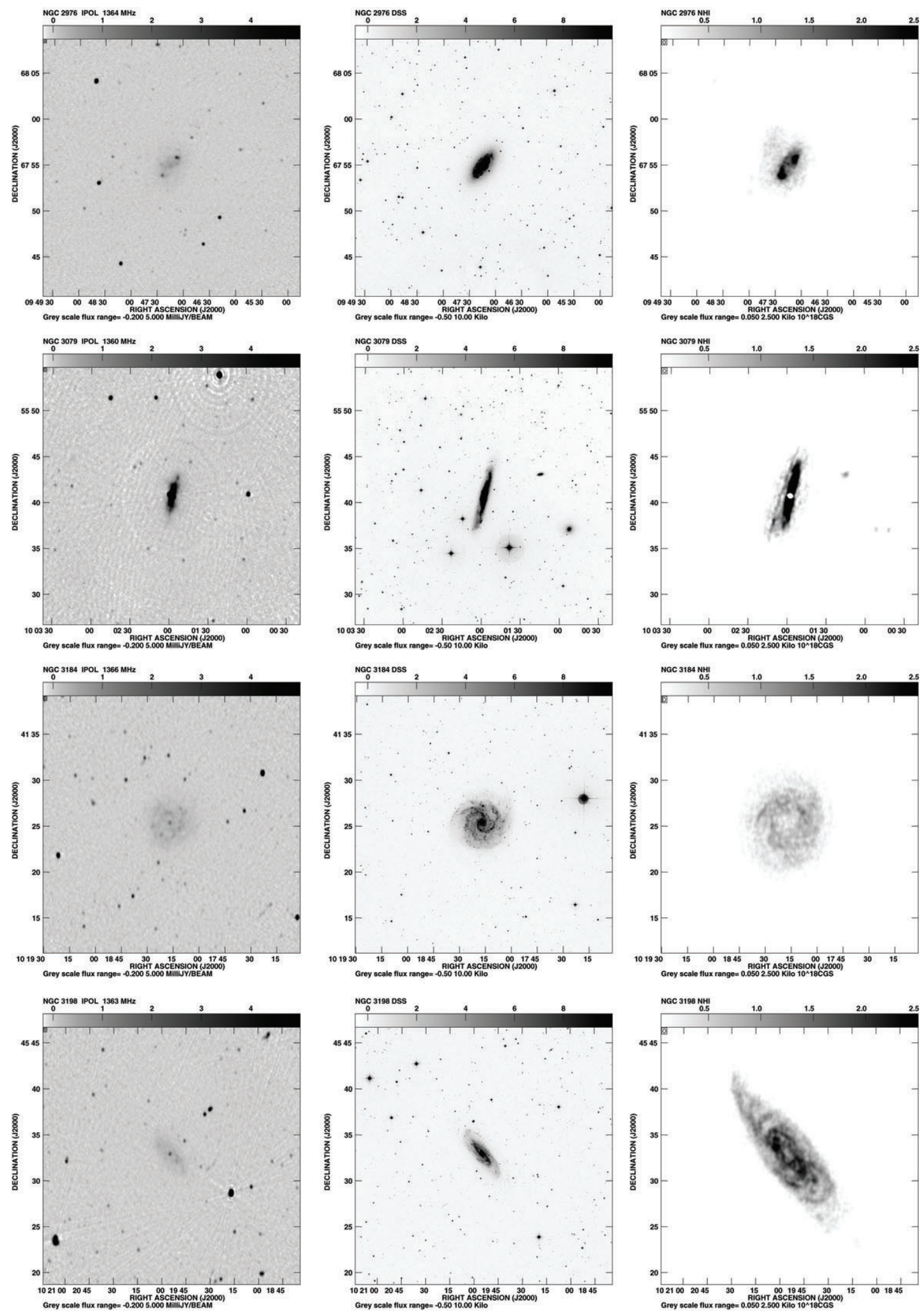

Fig. 4. Radio continuum (left), optical POSS-II Red (center) and neutral hydrogen column density (right) for the four indicated galaxies. A square root transfer function is used for the radio continuum image and a linear one for the optical (in densitometer counts) and HI images.

incomplete visibility sampling. We have marked the NGC 2403 and 5055 values with a ":" suffix in Table 2 to indicate the poor quality of these estimates. The effective velocity resolution in the $\mathrm{H}$ I line cubes is $132 \mathrm{~km} \mathrm{~s}^{-1}$, which is so coarse that significant sensitivity loss will be experienced for intrinsically narrow lines (such as the $25 \mathrm{~km} \mathrm{~s}^{-1} F W H M$ of a thermal $10^{4} \mathrm{~K}$ gas).

\subsection{Notes on individual galaxies}

Holmberg II Diffuse, low-level radio continuum emission is detected in the region of highest stellar surface density of this galaxy as well as several higher brightness features to the East. The work of Tongue \& Westphal (1995) shows that all of these 

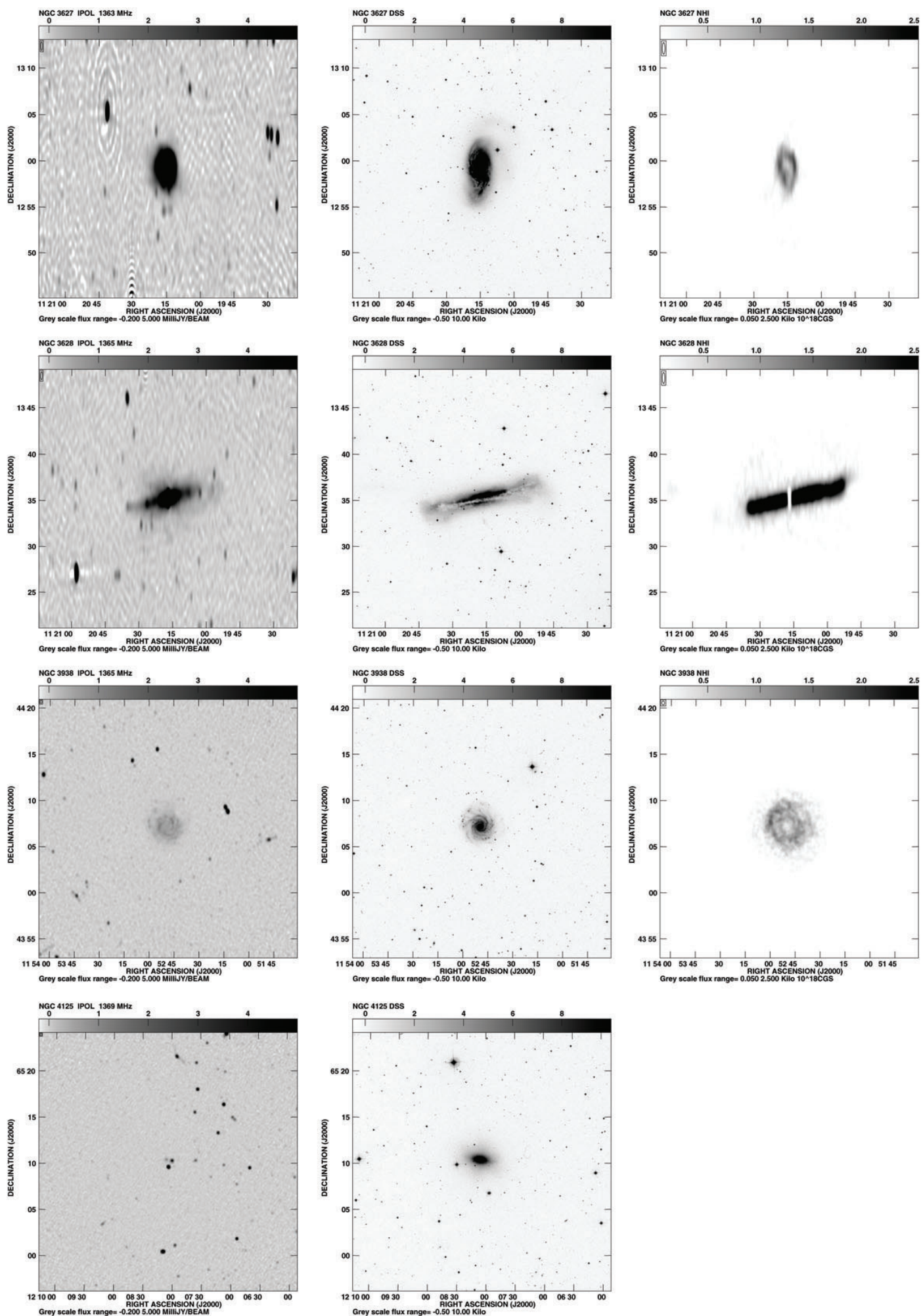

Fig. 5. Radio continuum (left), optical POSS-II Red (center) and neutral hydrogen column density (right) for the four indicated galaxies. A square root transfer function is used for the radio continuum image and a linear one for the optical (in densitometer counts) and HI images.

brighter features appear to be associated with $\mathrm{H} \alpha$-emitting regions; the fainter ones are likely to be purely thermal, while the brighter ones emit a mixture of free-free (from $\mathrm{H}$ II regions) and synchrotron (from supernovae or remnants) radiation. A classical double background radio galaxy of FR II type with an indication of precessing beams is seen in the South-East.
IC 2574 Diffuse radio continuum emission from the stellar disk is not detected convincingly. Brighter knots in the North-East are associated with regions of massive star formation in the galaxy. They embrace the small, but pronounced, $\mathrm{H}$ I hole in this galaxy adjoining the region of highest column density. This it is one of the few documented cases of an HI hole associated with a classical hot (X-ray emitting) bubble (Walter et al. 1998). 

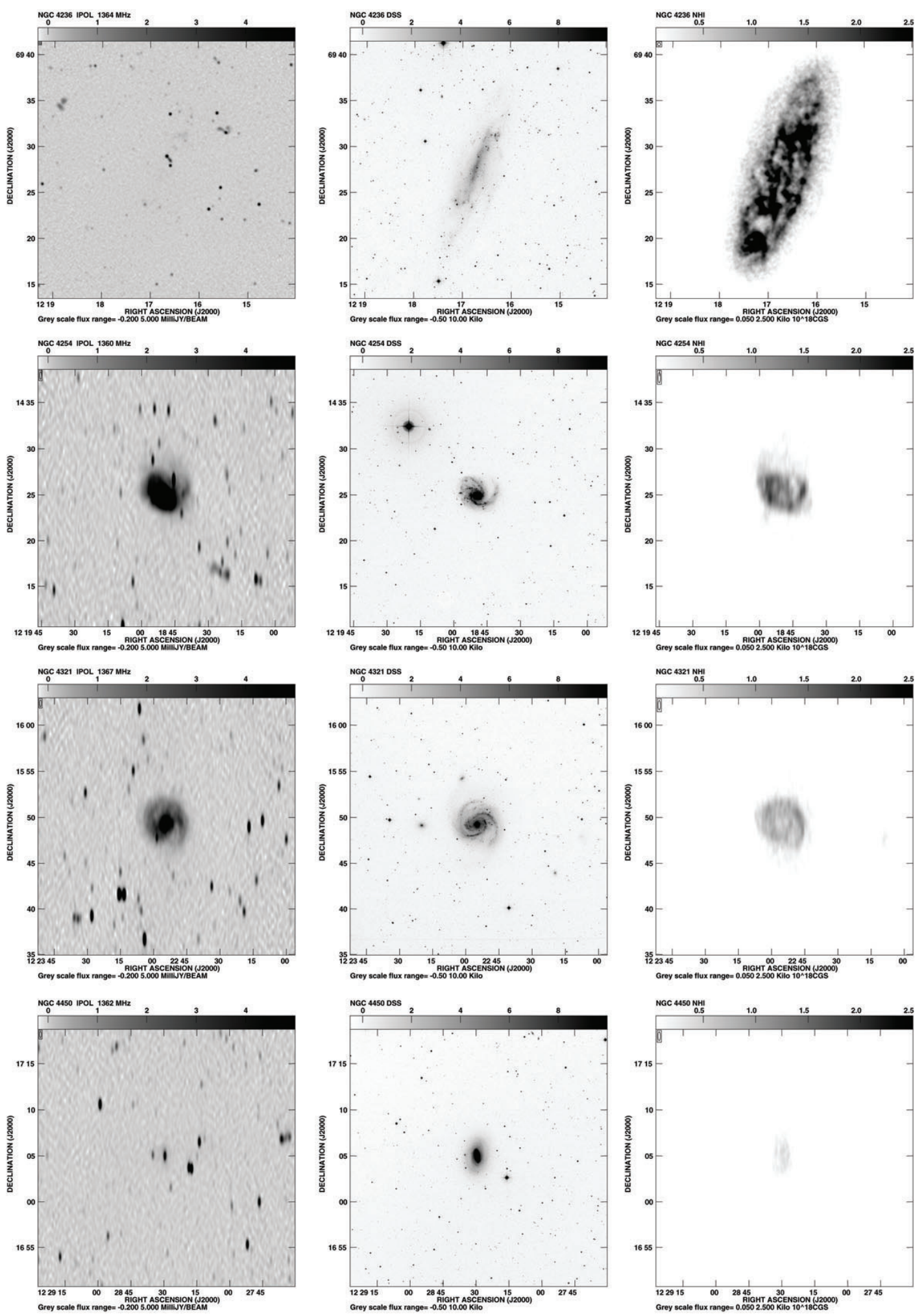

Fig. 6. Radio continuum (left), optical POSS-II Red (center) and neutral hydrogen column density (right) for the four indicated galaxies. A square root transfer function is used for the radio continuum image and a linear one for the optical (in densitometer counts) and HI images.

NGC 628 This galaxy possesses a very extended HI envelope, most likely fed by infalling intergalactic gas (Kamphius \& Briggs 1992). The radio continuum map shows diffuse disk emission as well as the two main spiral arms at higher surface brightness. Curiously, the optically bright central region is deficient in the radio continuum, although the $\mathrm{CO}$ map of Wakker $\&$ Adler (1995) suggests the presence of copious molecular gas within the central $30^{\prime \prime}$ region, albeit without any concentration towards the very center.

$N G C 925$ A low surface brightness disk is detected in this asymmetric late-type galaxy. The overall asymmetry may be attributed to infall and frequent gravitational encounters of the past few gigayears (Pisano et al. 1998). Yet the star formation density reflected by the radio continuum brightness is low. 

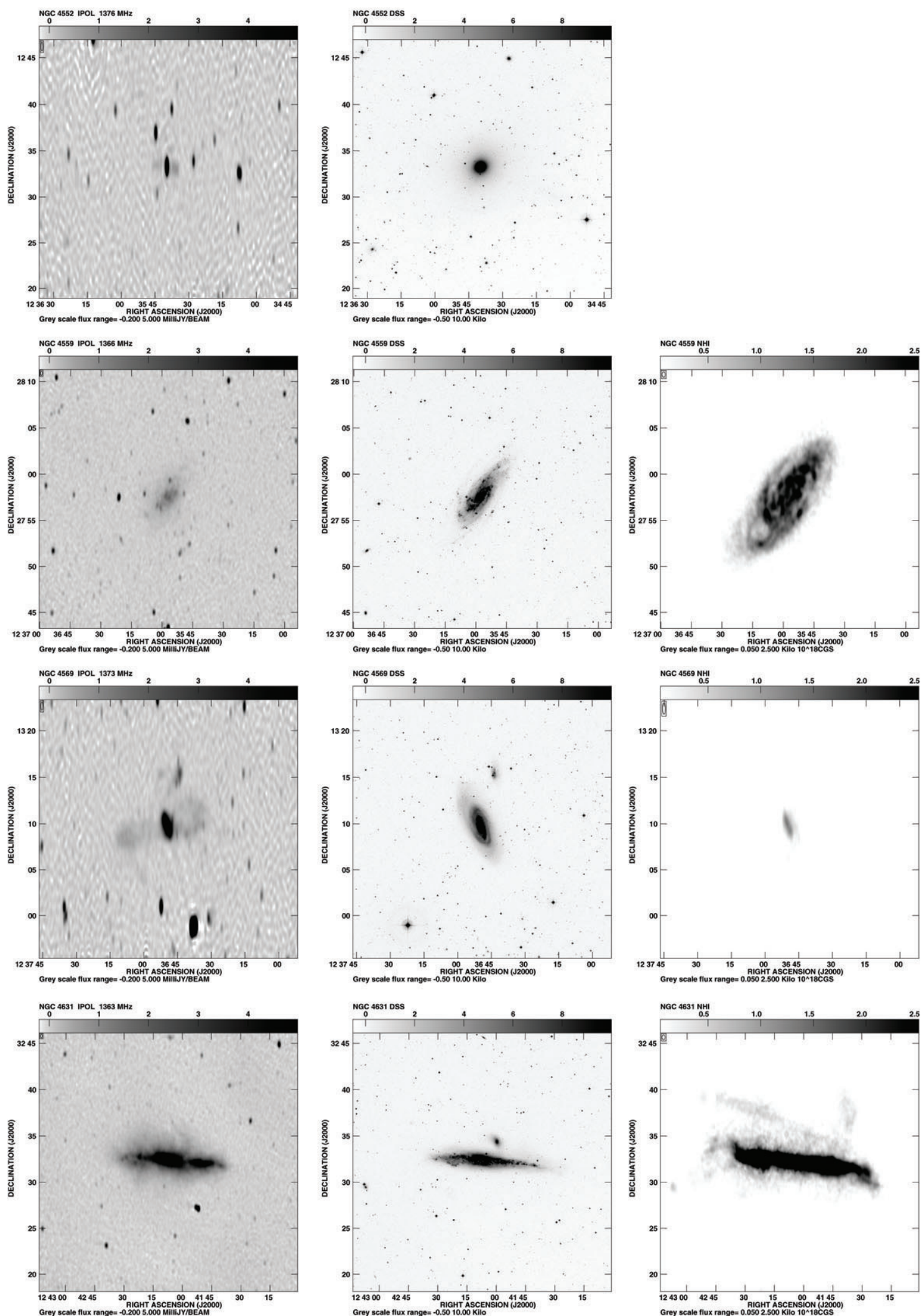

Fig. 7. Radio continuum (left), optical POSS-II Red (center) and neutral hydrogen column density (right) for the four indicated galaxies. A square root transfer function is used for the radio continuum image and a linear one for the optical (in densitometer counts) and HI images.

NGC 2146 Residual calibration errors are responsible for the "spoke-like" artifacts radiating from the nuclear region. This galaxy is a prototypical merger, evidenced by tidal Hi structures and a warped optical disk. The streamer seen in the neutral hydrogen towards the South continues into a very extended tidal tail (Taramopoulos et al. 2001). The merger has resulted in vigorous star formation throughout this galaxy, resulting in a brightness comparable to that of M 82. Similar to 

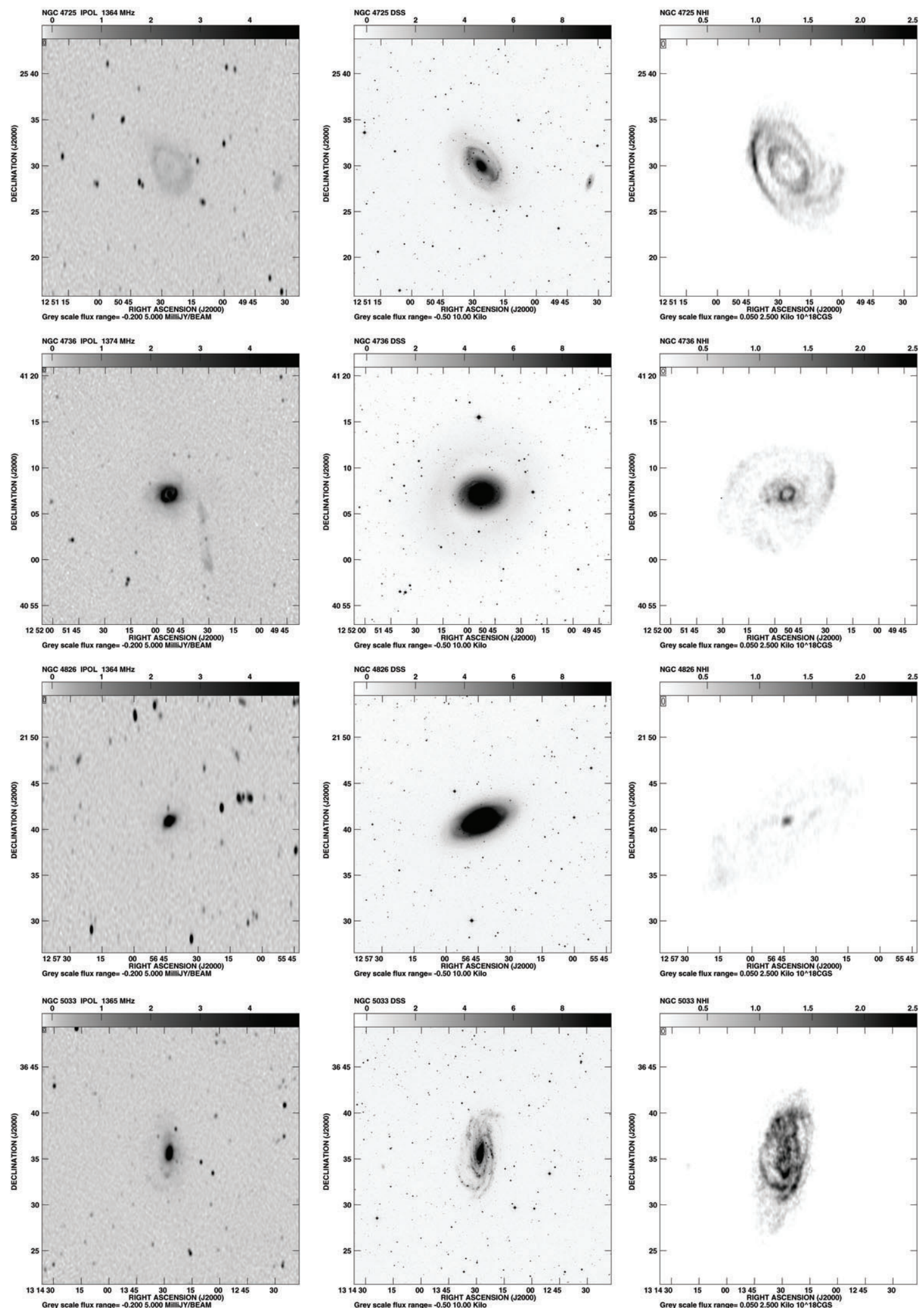

Fig. 8. Radio continuum (left), optical POSS-II Red (center) and neutral hydrogen column density (right) for the four indicated galaxies. A square root transfer function is used for the radio continuum image and a linear one for the optical (in densitometer counts) and $\mathrm{HI}$ images.

this nearby prototype, the bright radio continuum contains numerous compact sources reflecting ultra-compact $\mathrm{H}$ II regions and/or radio supernovae (Tarchi et al. 2000). They are also coincident with the prominent absorption feature seen in the H I map.
The $\mathrm{OH}$ main lines at 1667 and $1665 \mathrm{MHz}$ are detected in absorption against the nuclear region as shown in Fig. 11. Faint $1667 \mathrm{MHz}$ absorption may be detected at velocities blue-shifted by up to $200 \mathrm{~km} \mathrm{~s}^{-1}$ relative to the main disk. The peak line 

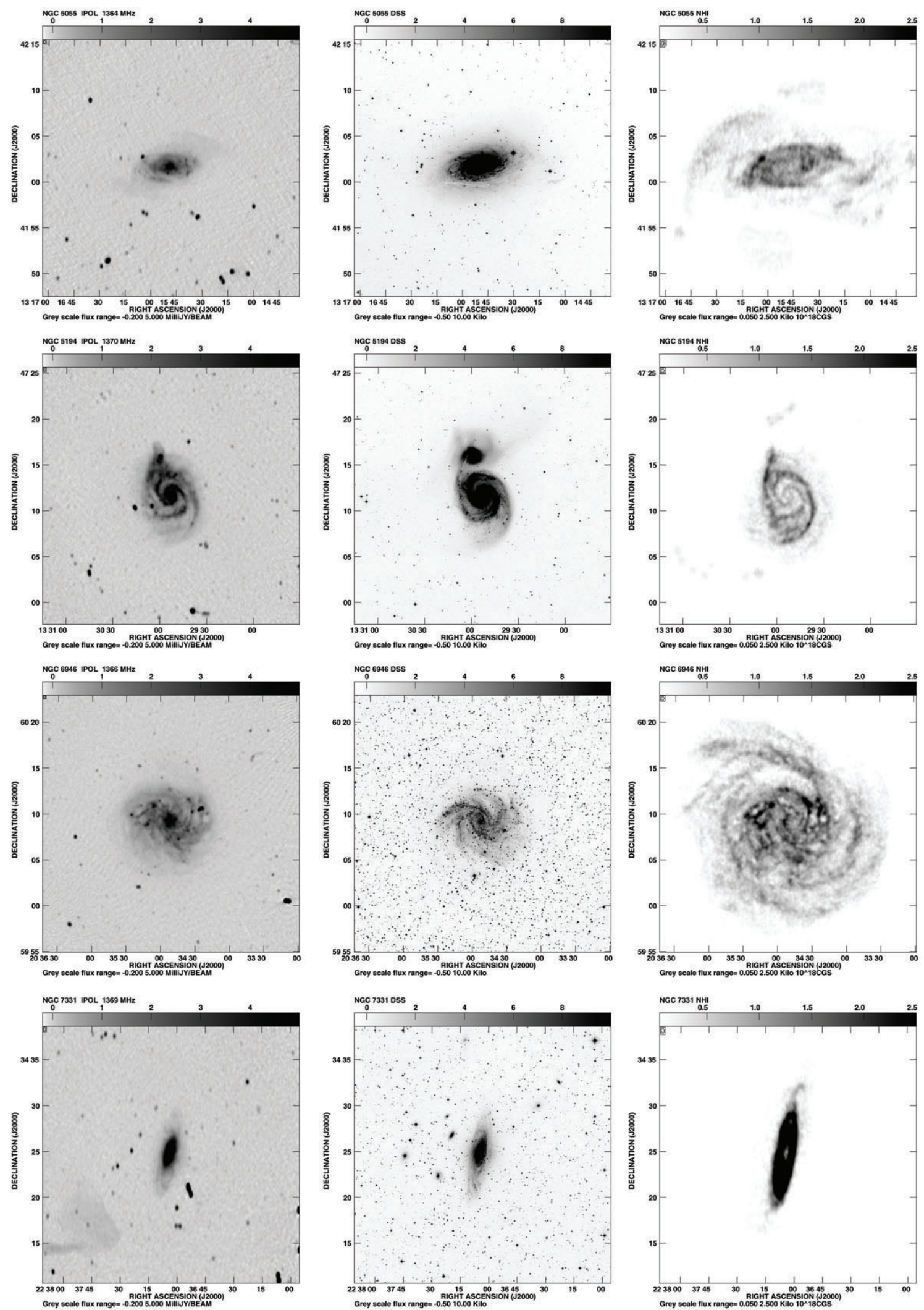

Fig. 9. Radio continuum (left), optical POSS-II Red (center) and neutral hydrogen column density (right) for the four indicated galaxies. A square root transfer function is used for the radio continuum image and a linear one for the optical (in densitometer counts) and HI images.

depths at 1667 and $1665 \mathrm{MHz}$ are -3.13 and $-1.70 \mathrm{mJy} /$ Beam relative to a continuum brightness of $387 \mathrm{mJy} /$ Beam measured with the same PSF. This $1667 / 1665$ line ratio correponds closely to the value of 1.8 expected from an optically thin thermal gas
(Heiles 1969). The H I absorption line depth is $-40.3 \mathrm{mJy} / \mathrm{Beam}$ relative to the $480 \mathrm{mJy} /$ Beam continuum brightness. The 10:1 observed ratio of $\mathrm{HI}$ to $\mathrm{OH}$ opacity is typical of that seen in the Galactic survey of Liszt \& Lucas (1996). 

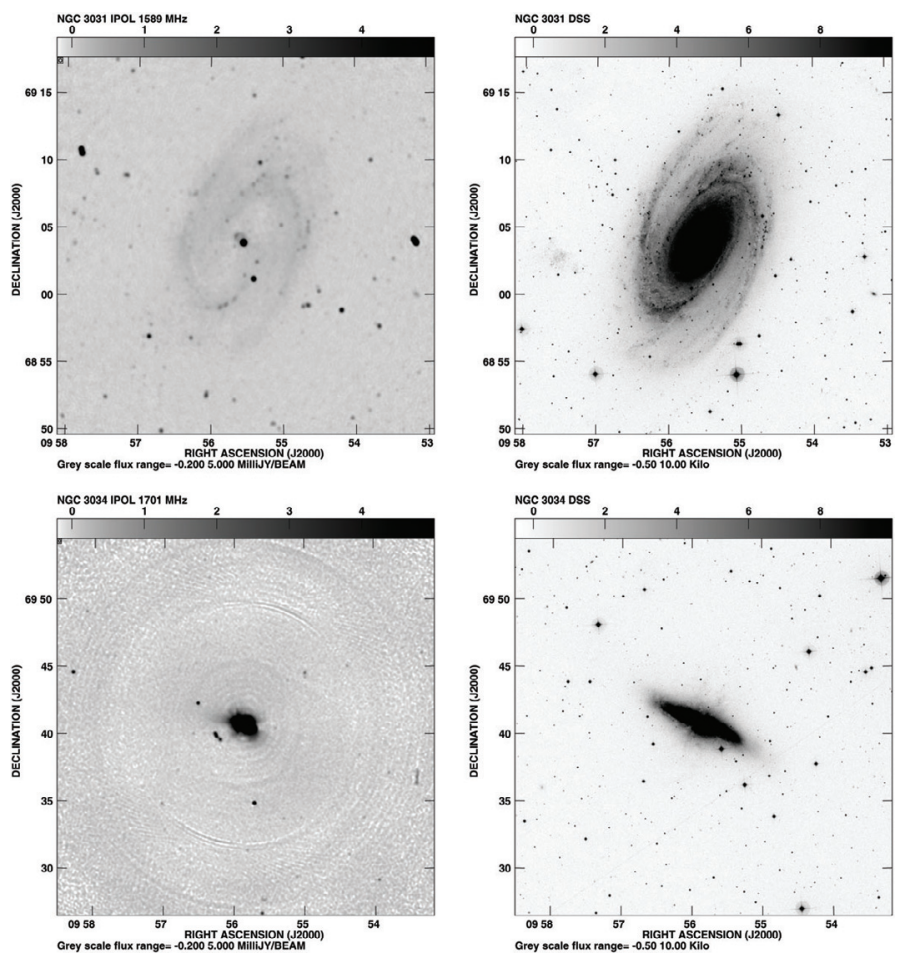

Fig. 10. Radio continuum (left) and optical POSS-II Red (right) images for the indicated galaxies. A square root transfer function is used for the radio continuum image and a linear one for the optical (in densitometer counts) image.

NGC 2403 This late-type spiral is a twin of M 33. The faint radio continuum from the disk has a number of brighter spots superimposed, which are coincident with prominent $\mathrm{H}$ II regions (Drissen et al. 1999). The galaxy has become known for the well-documented "anomalous" velocity gas component associated with the halo (Faternali et al. 2001, 2004) which may be the signature of accreting gas.

NGC 2841 A nuclear emission component is detected as well as a more diffuse hour-glass structure of $5^{\prime}$ major-axis diameter. The two brighter regions mark the apparent waist of this diffuse structure (see also Fig. 1 in Hummel \& Bosma 1982). This feature is reminiscent of a large diameter bi-polar outflow along the minor axis, but may well have some other origin. The H I map exhibits low column densities within the stellar disk, and extends well beyond this, but with a different position angle, indicative of a warp, as has been previously documented (e.g. Bosma 1981). $N G C 2903$ This is a grand-design spiral with a bright central region characterised by a starburst. A high-resolution study of the central region was performed by Saikia et al. 1994). The radio continuum, $\mathrm{H} \alpha$ and $\mathrm{CO}$ emission are almost identically distributed along a central bar (Jackson et al. 1991). The H I traces both the inner and outer spiral structures, but also extends far beyond the stellar disk, though with a different position angle. As in the above case, this is indicative of a warp (e.g. Wevers et al. 1986).

NGC 2976 Diffuse disk emission is seen in this dwarf spiral, together with brighter knots at either end of the major axis. These are coincident with strong $\mathrm{H} \alpha$ emission (see the images in Simon et al. 2003). This gives the same overall appearance in the radio continuum as seen in case of NGC 2841 and may also represent a similar phenomenon.

NGC 3031 This is the well known galaxy M 81, the dominant galaxy of the M 81 Group, and it is one of the best-studied spiral galaxies in all spectral regimes. Compared to other grand-design spirals, its radio continuum brightness is rather low. The overall spiral structure is readily recognisable in the radio continuum. The low radio continuum brightness and corresponding low star formation activity is in accord with the low CO surface brightness throughout M 81 (Brouillet et al. 1991; 1998). The central point source is a low-luminosity AGN (e.g. de Bruyn 1976; Filippenko 1988).

NGC 3034 As M 81's nearest companion, this prototypical dwarf starburst galaxy has seen countless studies, from radio wavelengths through gamma-ray energies. Similar to NGC 2146 (see above), although on a smaller scale, its violent star formation activity is caused by tidal interaction (in this case with M 81) and the resulting gas infall. Our radio continuum image delineates the central starburst, with some possible filaments emerging from the disturbed disk (although spoke-like calibration artifacts are also present), which were previously reported by Reuter et al. (1992). The double source to the South-East is a background radio galaxy.

NGC 3079 Well known for its central activity, this galaxy produces a bi-conical wind along the minor axis, although of limited angular extent. The corresponding "figure-eight" structure seen in the radio continuum on smaller scales (Duric \& Seaquist 1988) is not resolved in our 22-cm image, but is apparent as a protusion East of the nucleus. The H I map exhibits strong central absorption and two dwarf galaxies towards the West and North-West, which were previously reported by Irwin et al. (1987). Away from the plane, there are plumes of radio continuum emission in the North-East and in the South-West, while in the Hi line a linear structure is apparent, which was not visible in the lower-resolution VLA maps of Irwin et al. This feature is suggestive of tidal debris of a recent merger (cf. NGC 4631 below). OH mainline absorption was detected previously in this galaxy by Haschick \& Baan (1985). This is also seen prominently in Fig. 11. Peak line depths at 1667 and $1665 \mathrm{MHz}$ are -28.5 and $-22.4 \mathrm{mJy} /$ Beam relative to a continuum brightness of $398 \mathrm{mJy} /$ Beam measured with the same PSF. The 1667/1665 line ratio of 1.27 approaches the value of unity expected for an optically thick thermal gas (Heiles 1969). The H I absorption line depth is $-35.7 \mathrm{mJy} /$ Beam relative to the $486 \mathrm{mJy} /$ Beam continuum brightness. The 1:1 observed ratio of $\mathrm{H}$ I to $\mathrm{OH}$ opacity is more extreme in molecular content than any seen in the Galactic survey of Liszt \& Lucas 1996.

$N G C 3184$ Nuclear and faint disk emission are detected in this spiral galaxy, within the $\mathrm{H} \alpha$-emitting area (see image in Doane et al. 2004).

NGC 3198 Nuclear and faint disk emission are detected in this spiral galaxy, known for the seminal study of its distribution of dark matter (van Albada et al. 1985; Begeman 1989). The galaxy is surrounded by an extensive asymmetric H I envelope.

NGC 3627 This radio-bright galaxy belongs to the Leo triplet, together with NGC 3628 (see below) and NGC 3623 (see Zhang et al. 1993, for an overview). The bright radio continuum is co-extensive with the bright stellar disk as well as intense $\mathrm{H} \alpha$ (Chemin et al. 2003), CO (Regan et al. 1999), and FIR (Smith et al. 1994) emission. A multi-frequency radio continuum and polarisation study was presented by Soida et al. (2001). Weak $\mathrm{OH}$ mainline absorption is detected against the brightest central radio continuum feature (offset by some 20 arcsec to the NE of the nuclear position) an shown in Fig. 11. Peak line depths at 1667 and $1665 \mathrm{MHz}$ are -1.46 and $-0.93 \mathrm{mJy} /$ Beam relative to a continuum brightness of $31.6 \mathrm{mJy} /$ Beam measured with the same PSF. The $1667 / 1665$ line ratio of 1.6 is intermediate 
Table 2. Galaxy properties.

\begin{tabular}{lccccc}
\hline \hline Name & $\begin{array}{c}\text { 1365 MHz flux } \pm \text { error } \\
(\mathrm{mJy})\end{array}$ & $\begin{array}{c}\text { Aperture radius } \\
(\operatorname{arcsec})\end{array}$ & Method & $\begin{array}{c}\text { HI flux } \pm \text { error } \\
\left.(\mathrm{Jy}-\mathrm{km} \mathrm{s})^{-1}\right)\end{array}$ & $\begin{array}{c}\text { Peak } N_{\mathrm{HI}} \\
10^{21} \mathrm{~cm}^{-2}\end{array}$ \\
\hline Holmberg II & $5.5 \pm 2$ & 65 & Poly & $246 \pm 10$ & 3.08 \\
IC 2574 & $14 \pm 2$ & 200 & Poly & $413 \pm 20$ & 3.89 \\
NGC 0628 & $200 \pm 10$ & 450 & $\mathrm{Rad}$ & $381 \pm 15$ & 1.44 \\
NGC 0925 & $90 \pm 10$ & 450 & $\mathrm{Blk} / \mathrm{Rad}$ & $272 \pm 12$ & 3.58 \\
NGC 2146 & $1100 \pm 10$ & 200 & $\mathrm{Blk} / \mathrm{Rad}$ & $72.4 \pm 3$ & 6.33 \\
NGC 2403 & $360 \pm 30$ & 800 & $\mathrm{Blk} / \mathrm{Rad}$ & $1030:$ & 4.47 \\
NGC 2841 & $100 \pm 7$ & 400 & $\mathrm{Blk} / \mathrm{Rad}$ & $195 \pm 10$ & 1.89 \\
NGC 2903 & $460 \pm 10$ & 300 & $\mathrm{Blk} / \mathrm{Rad}$ & $232 \pm 10$ & 2.47 \\
NGC 2976 & $68 \pm 5$ & 250 & $\mathrm{Blk} / \mathrm{Rad}$ & $57.3 \pm 3$ & 3.85 \\
NGC 3079 & $890 \pm 10$ & 300 & $\mathrm{Blk} / \mathrm{Rad}$ & $102 \pm 5$ & 8.13 \\
NGC 3184 & $80 \pm 5$ & 300 & $\mathrm{Blk} / \mathrm{Rad}$ & $112 \pm 5$ & 1.47 \\
NGC 3198 & $49 \pm 5$ & 300 & $\mathrm{Blk} / \mathrm{Rad}$ & $220 \pm 10$ & 2.65 \\
NGC 3627 & $500 \pm 10$ & 350 & $\mathrm{Blk} / \mathrm{Rad}$ & $35.2 \pm 2$ & 1.85 \\
NGC 3628 & $590 \pm 10$ & 400 & $\mathrm{Blk} / \mathrm{Rad}$ & $240 \pm 10$ & 5.15 \\
NGC 3938 & $80 \pm 5$ & 250 & $\mathrm{Blk} / \mathrm{Rad}$ & $69.8 \pm 4$ & 1.64 \\
NGC 4125 & $1.9 \pm 0.2$ & 25 & $\mathrm{GFit}$ & $<1$ & $<0.1$ \\
NGC 4236 & $26 \pm 5$ & 600 & $\mathrm{Poly}$ & $619 \pm 30$ & 4.74 \\
NGC 4254 & $510 \pm 10$ & 300 & $\mathrm{Rad}$ & $83.5 \pm 4$ & 2.01 \\
NGC 4321 & $310 \pm 10$ & 250 & $\mathrm{Rad}$ & $57.2 \pm 3$ & 1.23 \\
NGC 4450 & $13 \pm 2$ & 100 & $\mathrm{Box}$ & $5.87 \pm 1$ & 0.44 \\
NGC 4552 & $93 \pm 5$ & 120 & $\mathrm{Box}$ & $<1$ & $<0.1$ \\
NGC 4559 & $110 \pm 10$ & 450 & $\mathrm{Rad}$ & $306 \pm 15$ & 3.15 \\
NGC 4569 & $170 \pm 10$ & 450 & $\mathrm{Blk} / \mathrm{Rad}$ & $8.52 \pm 1$ & 1.33 \\
NGC 4631 & $1290 \pm 10$ & 450 & $\mathrm{Rad}$ & $511 \pm 25$ & 10.97 \\
NGC 4725 & $100 \pm 10$ & 600 & $\mathrm{Blk} / \mathrm{Rad}$ & $136 \pm 6$ & 2.42 \\
NGC 4736 & $320 \pm 10$ & 400 & $\mathrm{Blk} / \mathrm{Rad}$ & $85.7 \pm 4$ & 2.00 \\
NGC 4826 & $110 \pm 10$ & 150 & $\mathrm{Rad}$ & $52.1 \pm 2$ & 1.69 \\
NGC 5033 & $240 \pm 10$ & 450 & $\mathrm{Rad}$ & $181 \pm 10$ & 2.78 \\
NGC 5055 & $450 \pm 10$ & 450 & $\mathrm{Rad}$ & $280:$ & 2.59 \\
NGC 5194 & $1420 \pm 10$ & 450 & $\mathrm{Rad}$ & $159 \pm 7$ & 2.19 \\
NGC 6946 & $1700 \pm 10$ & 600 & $\mathrm{Rad}$ & $680 \pm 35$ & 3.14 \\
NGC 7331 & $590 \pm 10$ & 500 & $\mathrm{Blk} / \mathrm{Rad}$ & $201 \pm 10$ & 4.59 \\
\hline & & & &
\end{tabular}

between the optically thin and optically thick cases. No corresponding $\mathrm{H}$ I absorption is detected.

NGC 3628 This on-going merger galaxy has been the target of numerous studies. As a member of the Leo triplet, it appears to have suffered most from the mutual interaction, its stellar disk being strongly warped. Its activity is manifest over the entire electromagnetic spectrum, from radio (Reuter et al. 1991) through X-rays (Dahlem et al. 1996). Despite the strongly elongated synthesised beam of the WSRT at this declination, significant diffuse radio continuum emission is seen to extend in the $z$-direction on either side of the disk, with some bright protusions marking the footpoints of the most active regions. Strong absorption in the HI map marks the location of the active nucleus. $\mathrm{OH}$ absorption has previously been detected in this galaxy by Rickard et al. 1982. This is clearly seen in Fig. 11. Peak line depths at 1667 and $1665 \mathrm{MHz}$ are -12.6 and $-8.28 \mathrm{mJy} /$ Beam relative to a continuum brightness of $202 \mathrm{mJy} / \mathrm{Beam}$ measured with the same PSF. The $1667 / 1665$ line ratio of 1.52 is intermediate between the optically thin and optically thick cases. The HI absorption line depth is $-18.9 \mathrm{mJy} /$ Beam relative to the $233 \mathrm{mJy} /$ Beam continuum brightness. The 1.3:1 ratio of observed $\mathrm{H}$ I to $\mathrm{OH}$ opacity is almost as extreme as that detected in NGC 3079.

NGC 3938 The stellar kinematics of this galaxy was studied by Bottema (1988) along with that of NGC 3198. We detect a diffuse disk and emission from spiral arms in the radio continuum which are also prominent in $\mathrm{H} \alpha$ (see the image in Jiménez-Vicente et al. (1999). The H I traced in our observations does not extend much beyond the stellar disk.
NGC 4125 This is an elliptical galaxy containing an incipient disk. According to Fabbiano \& Schweizer (1995) its rich finestructure (they report two plumes or disks crossing at right angels) is indicative of a recent merger. We detect a modest nuclear source coincident with the stellar galaxy nucleus and clearly separated from what appears to be a classical double radio galaxy displaced to the South-West.

NGC 4236 Diffuse disk emission is detected, together with knots of enhanced brightness in this member of the M 81 group (like NGC 2403, see above). The two brightest radio sources in the field center (a double and single source) are likely to be background objects. Despite the abundant (atomic) hydrogen, its star formation rate is low, with only very diffuse radio emission detectable.

NGC 4254 This galaxy is at the periphery of the Virgo Cluster. Its structure and kinematics were recently studied by Vollmer et al. (2000); its radio polarisation and magnetic field structure were investigated by Soida et al. (1996). Its strongly asymmetric structure is evident in both the radio continuum and the $\mathrm{H} \mathrm{I}$, with faint extensions to the North that go well beyond the optical disk.

$N G C 4321$ This classical grand-design spiral, also located in the Virgo Cluster, has been the target of numerous studies, e.g. in the radio continuum (van der Hulst 1981), H I (Knapen et al. 1993), and in CO (Cepa et al. 1992). The rather elongated synthesised beam reduces contrast in our radio continuum map. The $\mathrm{HI}$ emission is again (as in other Virgo spirals) truncated near the edge of the optical disk. 

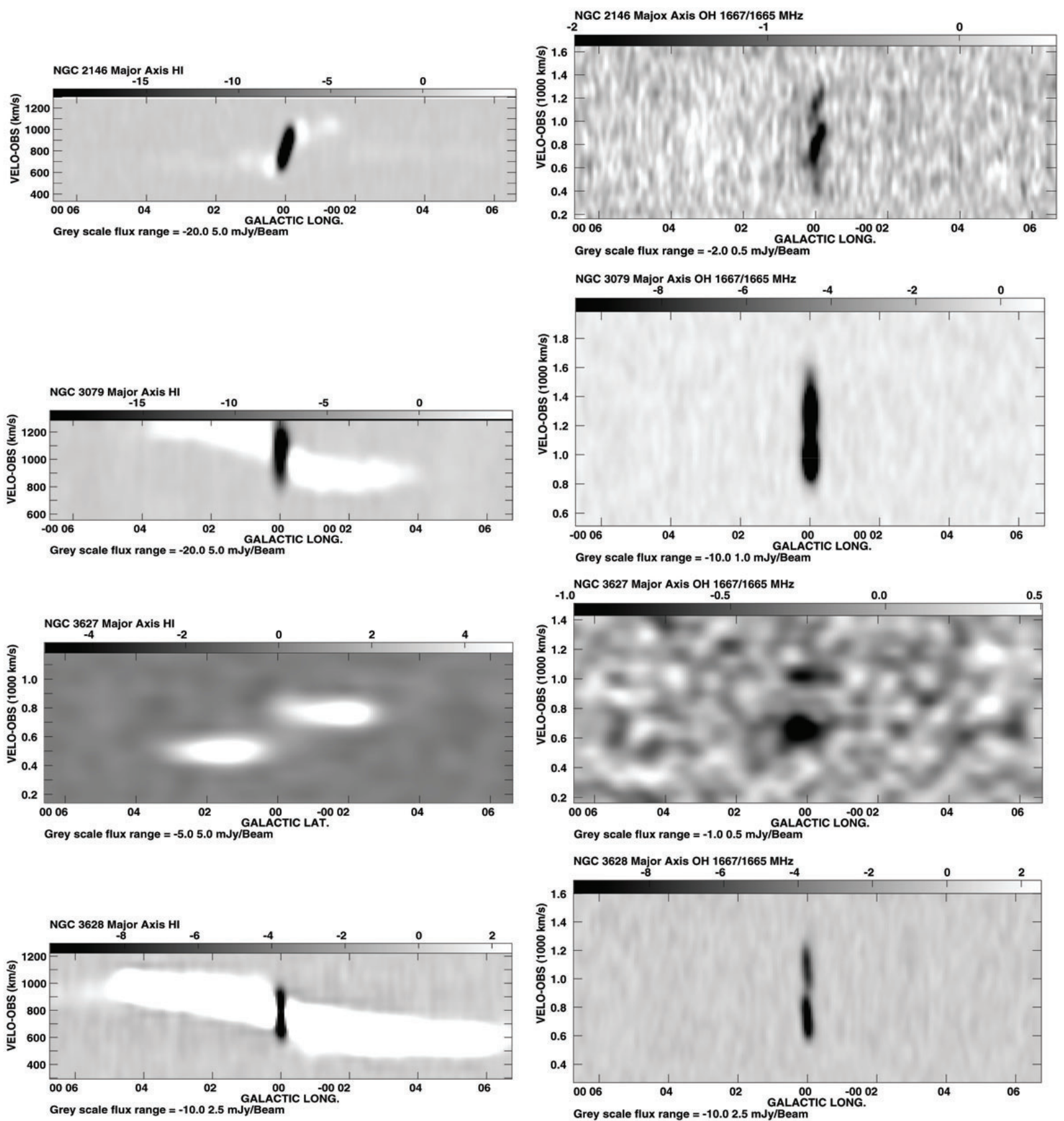

Fig. 11. HI emission and absorption (left) and $\mathrm{OH} 1667 / 1665 \mathrm{MHz}$ absorption (right) along the major axis of the indicated galaxies. A linear transfer function is used with the indicated range.

NGC 4450 Another member of the Virgo Cluster. Nuclear emission and a disk with small scale-length are detected in the radio continuum map of this LINER galaxy. It exhibits little H I.

NGC 4552 This is an elliptical galaxy belonging to "Subcluster A", located East of the Virgo Cluster (see Machacek et al. 2006. Its low radial velocity implies that it is moving supersonically through the Virgo Cluster, thus making it a prime candidate for (former) ram-pressure stripping. Our radio continuum map is the first obtained for this galaxy and shows nuclear emission and a double lobe along $\mathrm{PA} \sim 80^{\circ}$. Such a double lobe is suggestive of previous activity from what is now a low power AGN in the centre of this galaxy (Cappellari et al. 1999).

NGC 4559 Diffuse disk emisison is detected in this isolated spiral galaxy. Barbieri et al. (2005) have recently investigated its structure and kinematics in the $\mathrm{H}$ I line.
NGC 4569 A bright radio continuum disk with a small scalelength is detected co-aligned with the stellar component. The most salient feature is a double lobe extension along the minor axis at $\mathrm{PA} \sim 100^{\circ}$. There are but very few spiral galaxies of this kind, namely 0421+0400 (Beichman et al. 1988), 0313-192 (Keel et al. 2002), NGC 2992 (Wehrle \& Morris 1988), NGC 3079 (s.a.), NGC 3367 (García-Barreto et al. 1998), and NGC 4258 (e.g. van Albada \& van der Hulst 1982). They all share the common property of being radio-overluminous in the radio-FIR correlation.

NGC 4631 This edge-on galaxy is well known for its radio halo, which is also prominent in our radio continuum map. Numerous filaments extending from the disk into the halo are apparent. The H I map shows tidal features caused by the strong interaction with the nearby galaxy NGC 4656 (see the work of Rand 1994). 
NGC 4725 A low surface brightness ring and inner disk are detected in this galaxy. The ring is coincident with the star-forming ring for which this galaxy is known (Buta 1988). Its interaction with nearby NGC 4747 (Wevers et al. 1984) may be responsible for the asymmetries seen in our images.

$N G C 4736$ The kinematics and star formation activity in the central bar and ring of this LINER galaxy have been the focus of numerous investigations (e.g. Muñoz-Tuñón et al. (2004). We detect a high-surface brightness nuclear region, the inner ring and diffuse outer structures. Note the large ( $\left.7^{\prime}\right)$ background FR-II radio galaxy to the South-West.

NGC 4826 Also known as the "Black Eye" or "Evil Eye galaxy", this LINER exhibits a counter-rotating gaseous disk in its central region (see e.g. Braun et al. 1994; García-Burillo et al. 2003). The high column density $\mathrm{H}$ I in the central regions rotates in one sense, while the diffuse outer H I disk in the other. Our radio continuum image shows a high-surface brightness inner disk in this system.

NGC 5033 This tidally distorted Seyfert-I galaxy has seen numerous kinematic studies (e.g. Thean et al. 1997). Our H I map shows substantial asymmetry, the gaseous disk being strongly warped. The radio continuum image reveals a bright inner- and faint outer disk.

NGC 5055 This flocculent spiral galaxy possesses a huge $\mathrm{H}$ I disk (of $\sim 30$ arcmin diameter), which is strongly warped at large radii (Battaglia et al. 2006). The diffuse outer disk is not accurately represented in our $\mathrm{H}$ I image and the reader is referred to Battaglia et al. (2006) for a better representation. The radio continuum closely traces the stellar disk and its spiral structure. Two diffuse arcs are apparent to the North-West and South-East of the high brightness disk.

NGC 5194 Easily recognisable as "M 51" in any spectral regime, this prototypical grand-design spiral is one of the most thoroughly studied. Qualitatively, the radio continuum image is very similar to the optical (excepting the tidal companion NGC 5195), the high brightness regions are co-located with the regions of high star formation rate.

$N G C 6946$ As was the case for M 51 above, the radio continuum readily discloses the identity of this spiral, which has also been the target of numerous studies in all spectral bands. The H I extends far beyond the stellar disk, maintaining spiral structure as far out as it can be traced.

$N G C 7331$ A high-surface brightness inner disk is superposed on more diffuse outer arms and disk emission. Its high starforming activity may be connected with the counter-rotation of the bulge discovered by Prada et al. (1996). The structure of the galaxy is well illustrated in the recent work of Regan et al. (2004). The diffuse radio continuum in the South-East corner of the field is one lobe of a giant $\left(20^{\prime}\right)$ head-tail background radio galaxy.

\subsection{Final comments}

The most useful contribution of the data presented in this paper to the literature on nearby galaxies is to place their radio continuum emission in a more general context. Our survey has allowed resolved detection and imaging of every one of our sub-sample galaxies. The standardized presentation of Figs. 2-10 allows direct comparison of galaxies which span a remarkable range of morphological type and star formation rate; much as the Hubble Atlas (Sandage 1984) has allowed generations of astronomers to appreciate the diverse optical continuum appearance of nearby galaxies. A similar rich diversity of radio continuum (and for that matter $\mathrm{HI}$ ) appearances becomes obvious in the figures.
Although in this paper we have focused on the presentation of the main products (radio continuum and H I total intensity images) derived from the WSRT observations, the data have the potential to allow more follow-up and to help address some key questions:

- Star formation in a wide range of nearby galaxy environments: Radio continuum emission is closely tied to massive star formation (and death) or nuclear activity. It traces both the prompt (5 Myr) thermal emission from H II regions as well as the longer-lived ( $30 \mathrm{Myr})$ synchrotron emission of relativistic electrons in the local magnetic field. Previous studies have shown that at $\sim 1.5 \mathrm{GHz}$ radio frequencies the non-thermal:thermal ratio of galaxy disk emission is about 4:1 (e.g. Condon 1992), while the AGN contribution in most of our sample galaxies is seen to be minor. Hence the images are likely to be dominated by the non-thermal continuum emission of several $\mathrm{GeV}$ cosmic ray electrons.

As shown in Murphy et al. (2006) the Spitzer Infrared Nearby Galaxies Survey (SINGS; Kennicutt et al. 2003) and the WSRT-SINGS radio continuum survey presented here, can be used to study the effects of star-formation activity on the far-infrared (FIR)-radio correlation within galaxies. Future modeling of the physical mechanisms underlying the FIR-radio continuum correlation will certainly benefit from taking explicit account of the distinct timescales of all of the different emission mechanisms. These and many other topics will be addressed in subsequent papers utilizing the multispectral SINGS data.

- Galactic Magnetic Fields: a key component of the ISM is the magnetic field but in order to obtain as realistic picture as possible of the morphology and strength of the magnetic field in galxies, it is mandatory to map the nonthermal emission in such a way that a rotation measure (RM) analysis is rendered feasible. By using all of the individual frequency channels in each in the eight available sub-bands it is possible to trace continuous variations of the RM (see for a complete discussion Brentjens \& de Bruyn 2005).

The polarization characteristics of the galaxies in the sample, will be presented in a forthcoming paper.

- HI and $\mathrm{OH}$ emission/absorption: it is important to stress again the power of a wide-band, high resolution spectral line correlator such as currently available at the WSRT. In this way, we have been able to extract both the HI content and the presence (or absence) of $\mathrm{OH}$ absorption, in addition to the radio continuum morphology which was the main goal of the observations. Although not yet analyzed for the presented dataset, this has also enabled an unbiased survey of red-shifted emission line objects which fall in the band (as shown in Morganti et al. 2004).

Acknowledgements. The Westerbork Synthesis Radio Telescope is operated by ASTRON (Netherlands Foundation for Research in Astronomy) with support from the Netherlands Foundation for Scientific Research (NWO). The Digitized Sky Surveys were produced at the Space Telescope Science Institute under US Government grant NAG W-2166. The images of these surveys are based on photographic data obtained using the Oschin Schmidt Telescope on Palomar Mountain and the UK Schmidt Telescope.

\section{References}

van Albada, G. D., \& van der Hulst, J. M. 1982, A\&A, 115, 263 van Albada, G. D., Bahcall, J. N., Begeman, K., \& Sancisi, R. 1985, A\&A, 295, 305

Battaglia, G., Fraternali, F., Oosterloo, T., \& Sancisi, R. 2006, A\&A, 447, 49 Begeman, K. G. 1989, A\&A, 223, 47 
Bottema, R. 1988, A\&A, 197, 105

Buta, R. 1988, ApJS, 66, 233

Barbieri, C. V., Fraternali, F., Oosterloo, T., et al. 2005, A\&A, 439, 947

Beichman, C., Wynn-Williams, C. G., Lonsdale, C. J., et al. 1988, ApJ, 293, 148

Bosma, A. 1981, AJ, 86, 1791

Braine, J., Combes, F., Casoli, F., et al. 1993, A\&AS, 97, 887

Braun, R., Walterbos, R. A. M., Kennicutt, R. C., \& Tacconi, L. 1994, ApJ, 420, 558

Brentjens, M. A., \& de Bruyn, A. G. 2005, A\&A, 441, 931

Brouillet, N., Baudry, A., Combes, F., et al. 1991, A\&A, 242, 35

Brouillet, N., Kaufman, M., Combes, F., et al. 1998, A\&A, 333, 92

de Bruyn, A. G., Crane, P. C., Price, R. M., \& Carlson, J. B. 1976, A\&A, 46, 243

Cappellari, M., Renzini, A., Greggio, L., et al. 1999, ApJ, 519, 117

Cepa, J., Beckman, J. E., Knapen, J., et al. 1992, AJ, 103, 429

Chemin, L., Cayatte, V., Balkowski, C., et al. 2003, A\&A, 405, 89

Condon, J. J. 1992, ARA\&A, 30, 575

Dahlem, M., Heckman, T. M., Fabbiano, G., et al. 1996, ApJ, 461, 724

Doane, N. E., Sanders, W. T., Wilcots, E. M., \& Juda, M. 1999, AJ, 128, 2712

Drissen, L., Roy, J.-R., Moffat, A. F. J., \& Shara, M. M. 1999, AJ, 117, 1249

Duric, N., \& Seaquist, E. R. 1988, ApJ, 326, 574

Fabbiano, G., \& Schweizer, F. 1995, ApJ, 447, 572

Filippenko, A. V., \& Sargent, W. L. W. 1988, ApJ, 324, 134

Fraternali, F., Oosterloo, T., Sancisi, R., \& van Moorsel, G. 2001, ApJ, 562, L47

Fraternali, F., Oosterloo, T., \& Sancisi, R. 2004, A\&A, 424, 485

García-Barreto, J. A., Rudnick, L., Franco, J., \& Martos, M. 1998, AJ, 116, 111

García-Burillo, S., Combes, F., Hunt, L. K., et al. 2003, A\&A, 407, 485

Greenawalt, B., Walterbos, R. A. M., Thilker, D., \& Hoopes, C. G. 1998, ApJ, 506,135

Haschick, A. D., \& Baan, W. A. 1985, Nature, 314, 144

Heiles, C. 1969, ApJ, 157, 123

van der Hulst, J. M., Crane, P. C., \& Keel, W. C. 1981, AJ, 86, 1175

Hummel, E., \& Bosma, A. 1982, AJ, 87, 242

Irwin, J. A., Seaquist, E. R., Taylor, A. R., \& Duric, N. 1987, ApJ, 313, L91

Jackson, J. M., Eckart, A., Cameron, M., et al. 1991, ApJ, 375, 105

Jiménez-Vicente, J., Battaner, E., Rozas, M., et al. 1999, A\&A, 342, 417

Kamphius, J., \& Briggs, F. 1992, A\&A, 253, 335

Keel, W. C., Ledlow, M. J., \& Owen, F. N. 2002, BAAS, 34, 1245
Kennicutt, R. C. 1998, ApJ, 498, 541

Kennicutt, R. C., Armus, L., Bendo, G., et al. 2003, PASP, 115, 928

Knapen, J., Cepa, J., Beckman, J. E., et al. 1993, ApJ, 416, 563

Liszt, H., \& Lucas, R. 1996, A\&A, 314, 917

Machacek, M., Jones, W. R., \& Nulsen, P. 2006, ApJ, 644, 155

Morganti, R., Garrett, M., Chapman, S., et al. 2004, A\&A, 424, 371

Muñoz-Tuñón, C., Caon, N., \& Aguerri, J. A. L. 2004, AJ, 127, 58

Murphy, E. J., Braun, R., Helou, G., et al. 2006, ApJ, 638, 157

Pisano, D. J., Wilcots, E. M., \& Elmegreen, B. G. 1998, AJ, 115, 975

Prada, P., Gutierrez, C. M., Peletier, R. F., \& McKeith, C. D. 1996, ApJ, 463, L5 Rand, R. J. 1994, A\&A, 285, 833

Regan, M. W., Sheth, K., Vogel, S. N., \& Stuart, N. 1999, ApJ, 526, 97

Regan, M. W., Thornley, M. D., Bendo, G. J., et al. 2004, ApJS, 154, 204

Reuter, H.-P., Krause, M., Wielebinski, R., \& Lesch, H. 1991, A\&A, 248, 12

Reuter, H.-P., Klein, U., Lesch, H., et al. 1992, A\&A, 256, 10

Rickard, L. J., Bania, T. M., \& Turner, B. E. 1982, ApJ, 252, 147

Rots, A. H. 1980, A\&AS, 41, 189

Saikia, D. J., Pedlar, A., Unger, S., \& Axon, D. J. 1994, MNRAS, 270, 46

Sandage, A. 1984, Hubble Atlas of Galaxies, Carnegie Inst. of Washington

Simon, J. D., Bolatto, A. D., Leroy, A., \& Blitz, L. 2003, ApJ, 596, 957

Smith, B. J., Harvey, P. M., Colome, C., et al. 1994, ApJ, 425, 91

Soida, M., Urbanik, M., \& Beck, R. 1996, A\&A, 312, 409

Soida, M., Urbanik, M., Beck, R., et al. 2001, A\&A, 378, 40

Taramopoulos, A., Payne, H., \& Briggs, F. H. 2001, A\&A, 365, 360

Tarchi, A., Neininger, N., Greve, A., et al. 2000, A\&A, 358, 95

Thean, A. H. C., Mundel, C. G., Pedlar, A., \& Nicholson, R. A. 1997, MNRAS, 290,15

Tongue, T. D., \& Westphal, D. J. 1995, AJ, 109, 2462

Vollmer, B., Huchtmeier, W., \& van Driel, W. 2005, A\&A, 439, 92

Walter, F., Kerp, J., Duric, N., Brinks, E., \& Klein, U. 1998, ApJ, 502, L147

Wakker, B. P., \& Adler, D. S. 1988, AJ, 109, 134

Wehrle, A., \& Morris, M. 1988, AJ, 95, 1689

Wevers, B. M. H. R., Appleton, P. N., Davies, R. D., \& Hart, L. 1984, A\&A, 140, 125

Wevers, B. M. H. R., van der Kruit, P. C., \& Allen, R. J. 1986, A\&AS, 66, 505

White, R. L., \& Becker, R. H. 1992, ApJS, 79, 331

Zhang, X., Wright, M., \& Alexander, P. 1993, ApJ, 418, 100 